UCRL-JC-125676

PREPRINT

\title{
Modeling of Ion Implantation and Diffusion in Si
}

M-J Caturla

T. Diaz de la Rubia

P.J. Bedrossian

This paper was prepared for submittal to the

1996 International Symposium on Materials Science Applications of Ion Beam Techniques

Seeheim, Germany

September 9-12, 1996

and

Materials Science Forum

September 1996

This is a preprint of a paper intended forpublication in a journal or proceedings. Since changes may be made before publication, this preprint is made available with the understanding that it will not be cited or reproduced without the permission of the author. 


\section{DISCLAIMER}

This document was prepared $\approx m$ acconnt of work sponsored by an agency of the United States Govermment. Neither the United States Government nor the University of California nor any of their employees, makes any warranty, express or implied, or assumes any legal liability or respenabilicy for the accuracy, completeness, or usefulness of any information, apparatus, product, or process disclosed, or represents that its use wouldnot infringe privately owned riebls. Reference herein to any specific commercial products, process, or service by trade name, trademark, manufacturer, or otherwise, does not necessarily constitute or imply its eadorsement, recommendation, or favoring by the United States Government or the Uaiversity of California. The views and opinions of authors expressed herein do not necessarily state or reflect those of the United States Government or the University of California, and shall not be used for advertising or product endorsenent parposes. 


\title{
Modeling of ion implantation and diffusion in $\mathrm{Si}$
}

\author{
M-J Caturla, T. Diaz de la Rubia and P. J. Bedrossian
}

Lawrence Livermore National Laboratory, Livermore CA-94550 U.S.A.

Keywords: ion implantation, diffusion, molecular dynamics, Monte Carlo.

\begin{abstract}
Classical molecular dynamics simulations are used to study the damage produced during the implantation of semiconductors with different ion masses and energies between 1-25 keV. The time scale for these type of simulations is only on the order of ns, and therefore problems like transient enhanced diffusion of dopants or formation of extended defects can not be studied with these models. Monte Carlo simulations, including as input the results obtained from molecular dynamics calculations, are used to extend the simulation time, and in particular, to study processes like ion implantation and defects diffusion in semiconductors. As an example we show results for diffusion of the damage produced by implantation of silicon with $5 \mathrm{keV} \mathrm{Xe}$ ions at low doses. The results of the simulations are compared with experiments in order to validate the model.
\end{abstract}

\section{Introduction}

Predictive models for IC technologies require of a good understanding of the processes involved during ion implantation and thermal annealing of semiconductors [1]. The damage produced during ion implantation can play an important role on the diffusion of dopants during high temperature treatments. In particular, one of the key problems in the development of ultrashallow junctions is transient enhanced diffusion $[2,3,4]$ of dopants during the activation stage. The defects produced during implantation interact with the dopants and induce their migration over long distances, changing the initial implantation profile. The industry trend towards a decreasing size of semiconductor devices requires predictive models. Consequently, there is a strong need for a physically based description of the damage level in the crystal lattice after implantation, as well as understanding the role of this damage in the subsequent diffusion of the dopants.

We can summarize the basic mechanisms in ion beam processes as damage production by individual ions, defect diffusion and damage accumulation. Despite the important effort devoted over the last few years to elucidate some of these problems, many unknowns still remain. One of the reasons for this lack of understanding is the many parameters involved in these processes, like ion mass, energy, dose, dose rate, temperature of the lattice and impurity levels or traps. Regarding damage accumulation, it is known experimentally that at sufficiently high doses ion implantation can induce amorphization of the sample [5]. The critical dose to reach 


\section{Molecular dynamics simulation: source for diffusion modeling}

The first stage of the damage produced by ion implantation is obtained using classical molecular dynamics calculations. In our case we use the Stillinger Weber potential [15] to describe the interaction between the $\mathrm{Si}$-Si atoms in the lattice. For the short range interaction we modified this potential by connecting the two body term to the Universal potential [16], as described by Gärtner et al [17]. The interaction between the energetic ions and the silicon atoms is described also by the Universal potential. In order to account for inelastic energy losses, we use the Lindhard model [18] for those atoms with energies higher than $1 \mathrm{eV}$. The cells used in these simulations contain on the order of $10^{6}$ atoms. The boundary layers of the cell are connected to a thermostat in order to dissipate the energy deposited by the ion. The simulations were performed in a CRAY T3D MPP parallel computer at LLNL. The code employs PVM message passing routiens [19] for the communication between processors.
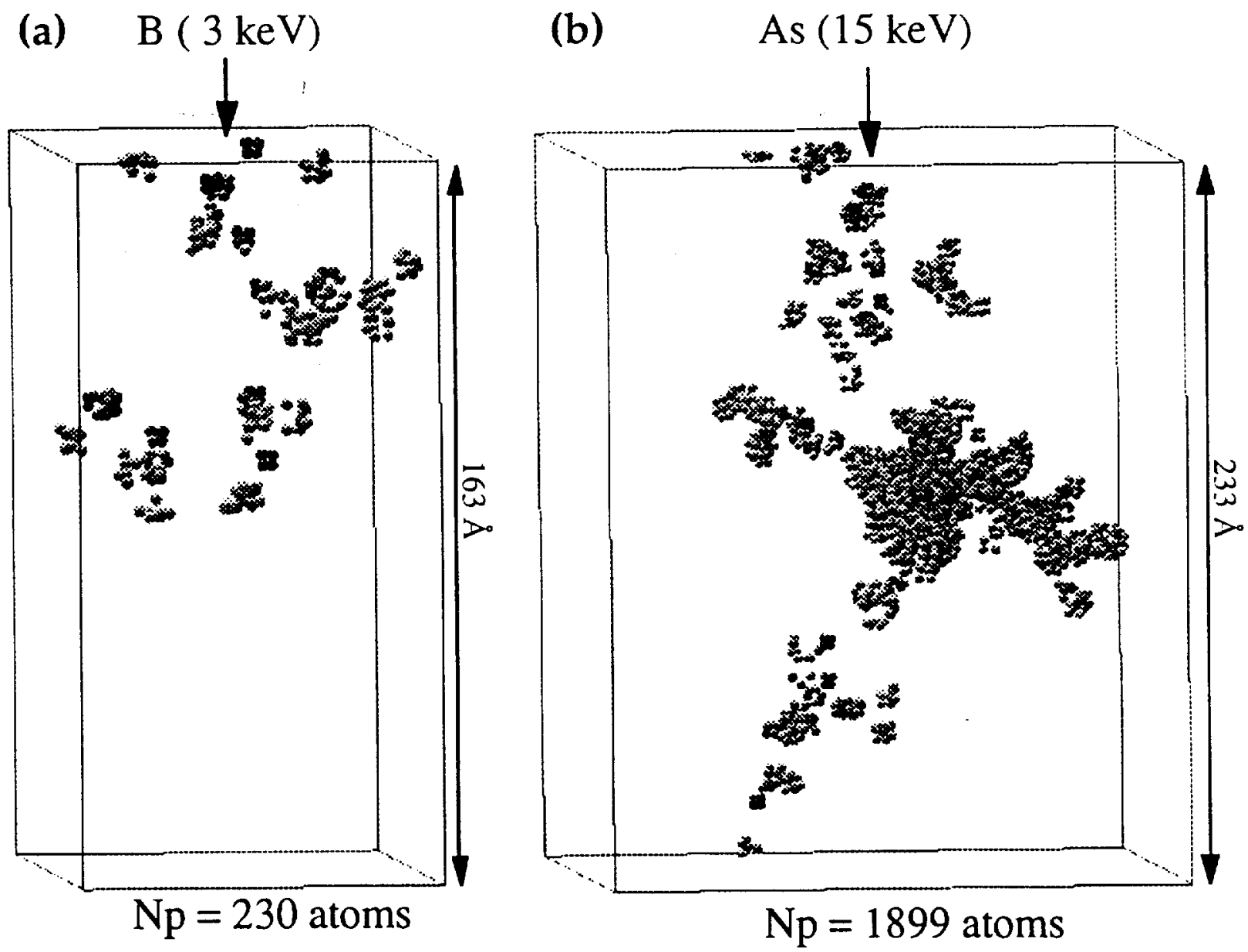

Figure 1. Molecular dynamics simualtion of ion implantation in Si for (a) $3 \mathrm{keV} \mathrm{B}$ irradiation and (b) $15 \mathrm{keV}$ As irradiation. Configuration of atoms with potential energy $>0.2 \mathrm{eV}$ with respect to the ground state, 10ps after the initil collision. 
amorphization depends on the ion mass, the temperature of the substrate and the ion flux $[6,7,8]$. Several models have been proposed to explain the amorphization mechanism, but none can give a complete description of all the experimental data available. One of the main difficulties is the dose rate dependence of amorphization, that is not taken into account in most of these models.

On the other hand, the diffusion kinetics of defects in silicon is also a controversial issue. For example, values for the migration energies of Vacancies (V) and Interstitials (I), as well as binding energies of clusters are not known experimentally, and discrepancies for interstitial diffusivity in Si as large as 9 order of magnitude at $700^{\circ} \mathrm{C}$ exist in the literature $[9,10]$. Recent molecular dynamics [11] and tight binding [12] simulations indicate lower migration energies for vacancies than for interstitials. The molecular dynamics simulations [11] also indicate lower binding energies for vacancy clusters with respect to interstitials. This poor understanding of the processes involved during semiconductor doping represent a major drawback in the development of predictive models. For a complete description of all these processes it is necessary to continue with the simulation efforts as well as develop new models in order to compare directly with the experimental results.

Molecular dynamics simulations have the advantage of giving a clear description about the microstructural processes occurring in the system under phenomenon like ion bombardment, since they describe the motion of all the atoms in the crystal [13]. However, they also involve large computational times. Even with the capabilities of parallel machines, only time scales on the order of nanoseconds can be achieved for the simulation of ion implantation. Processes like diffusion of defects under high temperature anneal, as in semiconductor doping, or amorphization processes, that depend on the ion flux can not be modeled using exclusively molecular dynamics. Recent simulations by M. Jaraiz [14] have shown that a good approach for the simulation of ion implantation and annealing at energies on the order of tens of $\mathrm{keV}$, consists on the coupling between binary collision approximation models, like MARLOWE, and Monte Carlo simulations. Following that approach, we use Monte Carlo simulations to study the annealing of defects at high temperatures, and extend the simulation time and scale. In our case the damage produced by the implantation, as well as the binding energies of the clusters of defects and the migration energies are obtained from molecular dynamics. This coupling between molecular dynamics and Monte Carlo allows us to simulate times on the order of seconds or longer, and therefore, validate our simulations by direct comparison with experiments.

In the next section we explain the molecular dynamics calculations performed in this work and how we use this data as input for the Monte Carlo simulation. The following section is a brief description of the Monte Carlo model, as well as the parameters used in these calculations. We finish with an example on how this tool can be applied to a real problem and compare with experimental results. 
In figure 1 we show the damage produced by a $3 \mathrm{keV}$ B ion, figure 1(a), as compared to a 15 $\mathrm{keV}$ As implantation, figure 1(b), 10ps after the implantation. These cases represent typical energies used in the production of shallow junctions, and correspond to the same range of the ions, approximately $130 \AA$. In this figure we show only those atoms that have a potential energy higher than $0.2 \mathrm{eV}$ with respect to the ground state in the perfect crystal. The grays represent the atomic level stress of the atoms, with dark grays being atoms under tension (and therefore, related to atoms surrounding a vacancy) and light grays representing those atoms under compression (mainly interstitials). It is clear from this picture that the morphology of the damage produced by these two ions is very different. While light ions only produce small clusters of defects, or isolated defects, heavier ions, like As, will produce large disordered zones. These zones have been reported earlier $[20,21]$ and have been proved to be amorphous in structure. This is also in agreement with experimental results for heavy ion implantation at low doses [22]. We can conclude from this first simulation that the damage induced during the doping of a semiconductor is going to depend on the type of dopant used, and in the same device we can have from small clusters of defects to localized amorphous pockets. All these effects have to be taken into account if we want to model a later evolution of the defects during a high temperature annealing.

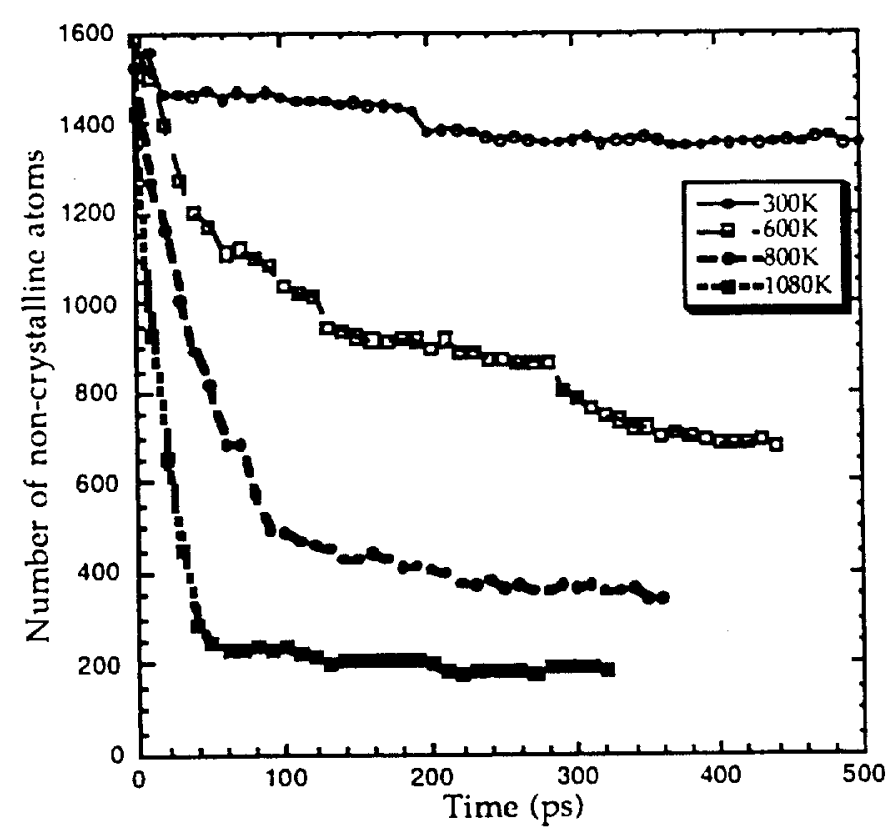

Figure 2. Annealing of the damage produced by 15 $\mathrm{keV}$ As implantation in $\mathrm{Si}$. Number of non-crystalline atoms as a function of annealing time, for different temperatures.

agreement with the recrystallization models proposed by Spaepen and Tumbull [23], and modified by Aziz [24] and Williams [25] for a planar amorphous-crystalline interface. According
The stability at different temperatures of the amorphous pockets produced by heavy ion bombardment is an important factor to understand damage accumulation during implantation. We have simulated the damage induced by the bombardment with $\mathrm{As}$ and $\mathrm{Pt}$ at energies between 5 and $25 \mathrm{keV}$ and studied the annealing kinetics of the amorphous pockets produced by these ions at different temperatures. In figure 2 we present the results for the number of amorphous atoms as a function of the annealing time and different temperatures for the case of the damage produced by the bombardment with a 15 $\mathrm{keV} \mathrm{As}$ ion. Observe that these curves do not follow an exponential behavior, instead there is an initial stage when recrystallization occurs in a very short annealing time. Later the recrystallization occurs by a series of plateaus and steps. This behavior is in 
to these models recrystallization occurs by the production and migration of dangling bonds at the interface. In our case, the irregular shape of the amorphous pockets provides the nucleation sites for recrystallization, as pointed out by Priolo [26], and recrystallization can occur at temperatures lower than in a planar interface.

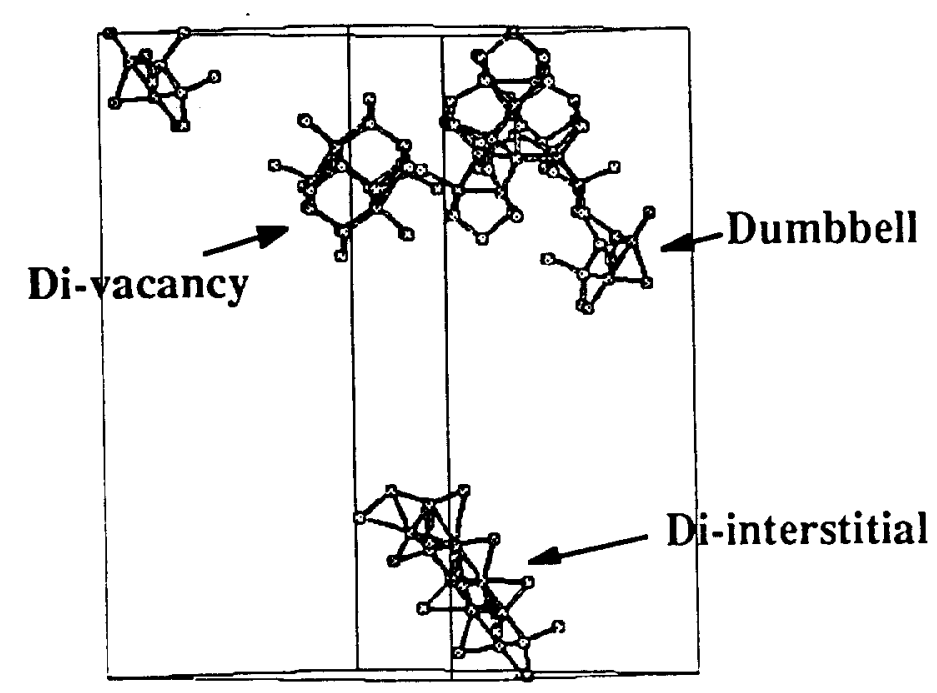

Figure 3. Defects at the Si lattice after $0.5 \mathrm{~ns}$ anneal at $800 \mathrm{~K}$. Only point defects and small clusters of defects are present.
Considering the results from figure 2 , at high temperatures the damage anneals in a very short time; after the first few picoseconds only point defects and small clusters of defects are left in the lattice. In figure 3 we show the defects present in the crystal after 0.5 ns anneal of the damage at $800 \mathrm{~K}$. From these results we can conclude that at high temperatures, like the ones used for the activation of the dopants in a semiconductor, only point defects and small clusters of defects are going to be present, and no local amorphization is left. Therefore, treatment of the diffusion of the defects at high temperatures and long times can be done using simple Monte Carlo simulations.

\section{Modeling of defect diffusion: Monte Carlo simulations}

Two types of defects are considered in the simulation, vacancies and interstitials. The initial positions of these defects are obtained from the molecular dynamics simulations as explained above. The values for binding energies of clusters of different sizes and migration energies are also obtained from molecular dynamics using the Stillinger Weber potential. These values are shown in Table I. The pre-factor for the self-interstitials has been obtained from fitting kinetic Monte Carlo results to the diffusion of boron in silicon [27] and using $a b$ initio results for the energetics of the boron-silicon interaction [28]. Clusters larger than 5 have binding energies fitted to a function of the form: $E b_{r} \cdot(N)=3.6-4.9\left(n^{2 / 3}-(n-1)^{2 / 3}\right) e V$ and $E b_{1}(n)=2.5-2.17\left(n^{1 / 2}-(n-\right.$ $\left.1)^{1 / 2}\right) \mathrm{eV}$, for $\mathrm{V}$ and $\mathrm{I}$ of size $n$ respectively. See reference [14] for a description of the program.

As an example of the capabilities of this simulation tool we consider the annealing at different temperatures of the damage produced by bombardment with $5 \mathrm{keV}$ Xe ions at a dose of $1.510^{13}$ ions $/ \mathrm{cm}^{2}$. The final dose is obtained by accumulating the damage induced by individual ions at room temperature. In this case we consider that there is no relaxation of the damage between cascades, approach based on the calculations explained above. The final kinetic-Monte Carlo simulation includes a total of 152 ion trajectories in a box of $0.32 \mathrm{mmx} 0.32 \mathrm{~mm} \times 5 \mathrm{~mm}$. 
Table I. Parameters used for the Monte Carlo simulation. Values for diffusivities and binding energies from classical molecular dynamics calculations [11]. The pre-factor for interstitials is obtained from fitting to boron diffusion experiments [27] and ab initio calculations [28].

\begin{tabular}{|c|c|c|c|c|c|}
\hline & DIFFUSIVTTY $\left(\mathrm{cm}^{2} / \mathrm{s}\right)$ & $\begin{array}{c}\text { BINDING } \\
\text { ENERGY } \\
\text { SIZE 2 (eV) }\end{array}$ & $\begin{array}{c}\text { BINDING } \\
\text { ENERGY } \\
\text { SIZE 3 (eV) }\end{array}$ & $\begin{array}{c}\text { BINDING } \\
\text { ENERGY } \\
\text { SIZE 4 (eV) }\end{array}$ & $\begin{array}{c}\text { BINDING } \\
\text { ENERGY } \\
\text { SIZE 5 (eV) }\end{array}$ \\
\hline VACANCY & $0.001 \mathrm{EXP}(-0.43 / \mathrm{KT})$ & 0.62 & 0.78 & 1.2 & 1.82 \\
\hline INTERSTITIAL & $5 . \operatorname{EXP}(-0.9 / \mathrm{KT})$ & 1.6 & 2.25 & 1.29 & 2.29 \\
\hline
\end{tabular}

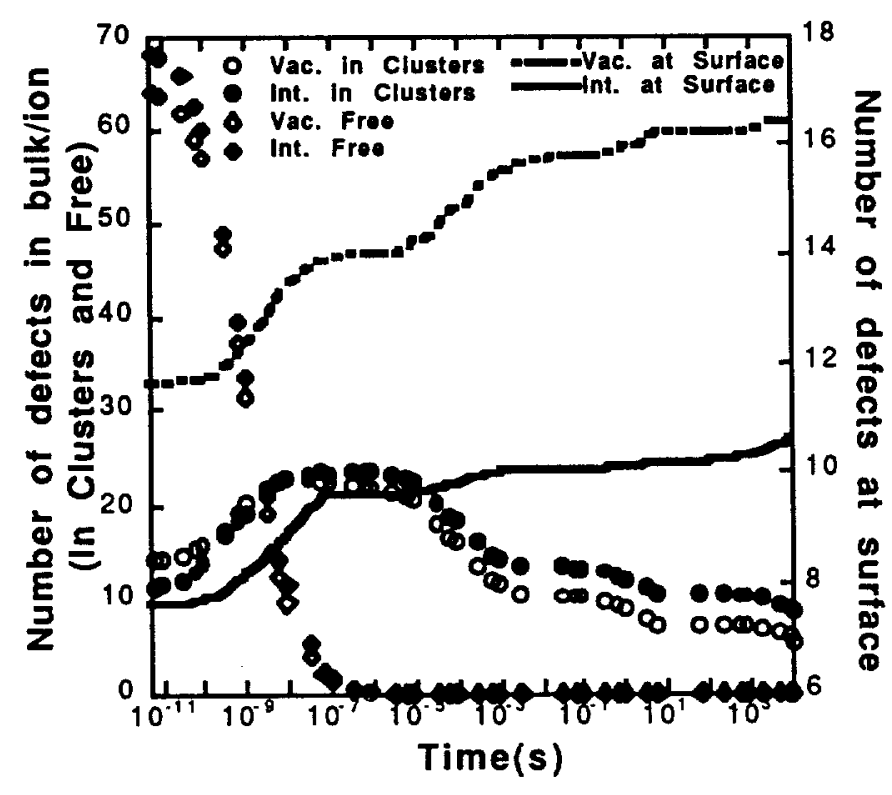

Figure 4. Kinetic Monte Carlo results for the annealing of the damage produced by $5 \mathrm{keV}$ Xe implantation at $1.5 \times 10^{13}$ ions $/ \mathrm{cm}^{2}$. Number of $V$ and $I$ in clusters and free (left hand side of the plot), as well as the number of defects at the surface (right hand side) as a function of the annealing time. Temperature of annealing is $350^{\circ} \mathrm{C}$.
First we study the evolution of the damage at low temperature, $350^{\circ} \mathrm{C}$, during a total annealing time of 4 hours. In figure 4 we show the number of free and clustered vacancies and interstitials in the bulk, as well as the number of vacancies and interstitials reaching the surface as a function of annealing time. During the initial stages of the anneal, when free defects are still present in the lattice, the diffusion is governed by the individual migration energies of vacancies and interstitials. With the parameters considered in this simulation the diffusivities for $V$ and $I$ at this temperature are very similar. Therefore these defects migrate and annihilate, recombine with the surface or form clusters of defects. After $10^{-7} \mathrm{~s}$ only clusters of defects remain in the bulk. Then diffusion is governed by the binding energies of the different clusters. In our case, the binding energies of vacancy clusters are always smaller than those of the interstitials. Therefore, the small vacancy clusters start releasing defects, and some of those will reach the surface. As a consequence the total number of vacancies reaching the surface after 4 hours of anneal is higher than the number of interstitials. Also the vacancy clusters increase in size, with an average cluster size of 6 for vacancies while the interstitial average cluster size is less than 3 . The sputtering yield obtained from the molecular dynamics simulations is of 2.25 atoms/ion consistent with experimental results [29]. After the 4 hours anneal at $350^{\circ} \mathrm{C}$ the excess number of vacancies at the surface is $\sim 6$ V/ion, and therefore, much higher than the sputtering yield. 
In figure 5 we show the results of a later annealing of the damage at higher temperature, $550^{\circ} \mathrm{C}$. In this case both vacancy and interstitial clusters dissociate. All the vacancies present in the bulk quickly disappear either on the surface or recombined with interstitials. Some of the interstitials also reach the surface, decreasing the excess number of vacancies per ion at the surface.

These results agree with experimental observations on $\mathrm{Si}(111)-7 \times 7$ [30]. In these experiments a depopulation of the adatom layer is observed under annealing at $350^{\circ} \mathrm{C}$, that could be explained as an arrival of vacancies to the surface from the bulk. The density of vacancies in the experiment is $1.2 \times 10^{14} \mathrm{~V} / \mathrm{cm}^{2}$, in reasonable agreement with the $0.8 \times 10^{14} \mathrm{~V} / \mathrm{cm}^{2}$ from our simulation. Annealing at $550^{\circ} \mathrm{C}$ induces repopulation of the surface layer, that considering our model, is due to the arrival of interstitials that evaporate from clusters in the bulk. In the experiment the density of interstitials at the surface after 2 minutes anneal is of $\sim 7 \times 10^{13} \mathrm{I} / \mathrm{cm}^{2}$ as compared to $\sim 3 \times 10^{13} \mathrm{I} / \mathrm{cm}^{2}$ obtained in the simulation.

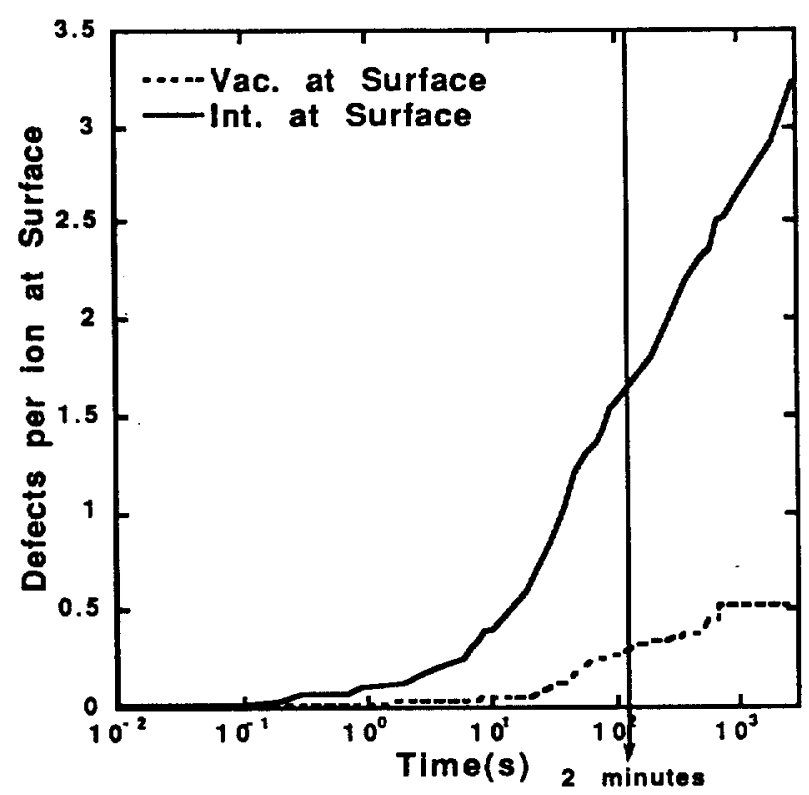

Figure 5. Number of defects per ion recombined at the surface as a function of the annealing time. The annealing temperature is $550^{\circ} \mathrm{C}$.

\section{Conclusions}

In summary, a combination of molecular dynamics and kinetic Monte Carlo simulations has been used to study ion implantation and diffusion in silicon. The time scale achieved with this type of simulations allows for direct comparison with experiments. In our case we apply the model to study the diffusion of defects produced during the irradiation of Si with $5 \mathrm{keV} \mathrm{Xe}$ ions, and the later diffusion at different temperatures. It is observed that, depending on the temperature, different defects reach the surface. At low temperatures an excess number of vacancies arrive to the surface. At higher temperatures, interstitial clusters dissolve decreasing the excess of vacancies at the surface. These results can explain the experimental observation of depopulation and repopulation of the $\mathrm{Si}$ (111)-7x7 surface at different annealing temperatures [30]. 


\section{Acknowledgments}

This work was performed under the auspices of the U.S. Department of Energy by Lawrence Livermore National Laboratory under Contract No. W-7405-Eng-48. M. Jaraiz and G.H. Gilmer of Lucent Technologies provided the KMC code. We thank L. Pelaz for useful discussions.

\section{References}

[1] J.D. Plummer, P.G. Griffin, Nucl. Instrum. Meth. B102 (1995) 160

[2] T. Motooka and O.W. Holland, Apply. Phys. Lett. 61 (1992) 3005

[3] K.S. Jones, H.G. Robinson, J. Listebarger, J. Chen, J. Liu, B. Herner, H. Park, M.E. Law, D. Sieloff, J.A. Slinkman, Nucl. Instrum. and Methods, B96 (1995) 196

[4] D.J. Eaglesham, P. A. Stock, HJ-J Gossman, and J.M. Poate, Appl. Phys. Lett. 65 (1994) 2305

[5] J.S. Williams, Mater. Res. Soc. Bull. 17, 47 (1992)

[6] R.G. Elliman, J. Linros, and W.L. Brown, Mater. Res. Soc. Synp. Proc. 100 (1988) p. 60

[7] P.J. Schultz, C. Jagadish, M. C. Ridgeway, R. G. Elliman and J.S. Williams, Phys. Rev. B44 (1991) 9118

[8] R.D. Goldberg, R.G. Elliman, and J. S. Williams, Nucl. Intrum. Meth. B80/81 (1993) 596

[9] H-J Gossmann, P.A. Stock, D.J. Eaglesham,. G. H. Gilmer and J.M. Poate, in Process

Physics and Modeling in Semiconductor Technology, edited by G.R. Srinivasan, D.S. Murthy and S.T. Dunham (Electrochemical Society, Pennington, New Jersey, 1996)

[10] U. Gösele, A. Plößl and T.,Y. Tan in Process Physics and Modeling in Seniconductor Technology, edited by G.R. Srinivasan, C.S. Murthy and S.T. Dunham (Electrochemical Society, Pennington, New Jersey, 1996) p.309

[11] G.H. Gilmer, T.Diaz de la Rubia, D. Stock and M. Jaraiz, Nucl. Instrum. and Methods B102 (1995) 247

[12] M. Tang, L. Colombo, J. Zhu, T. Diaz de la Rubia, Phys. Rev. B (accepted for publication)

[13] M.P. Allen and D.J. Tildesley in Computer simulation of liquids, Oxford science publications (1987)

[14] M. Jaraiz, G.H. Gilmer, J.M Poate, T. Diaz de la Rubia, Appl. Phys. Lett. 68 (1996) 409

[15] F.H. Stillinger and T.A. Weber, Phys. Rev. B31 (1985) 5262

[16] J.F. Ziegler, J.P. Biersack, U. Littmark, The stopping and Range of Ions in Solids, Vol.1 of The Stopping and Range of Ions in Matter, ed. J.F. Ziegler (Pergamon, New York, 1985) p. $25 \mathrm{ff}$ [17] K. Gärtner, Nucl. Instrum. and Methods, B102 (1995) 183

[18] J. Lindhard and M. Sharff, Phys. Rev. 124 (1961) 128

[19] PIM3 Users Guide and Reference Mamal. ORNL/TM-12187, May 1993

[20] T. Diaz de la Rubia, G.H. Gilmer, Phys. Rev. Lett. 74 (1995) 2507

[21] M-J Caturla, L. A. Marques, T. Diaz de la Rubia and G.H. Gilmer, Phys. Rev. B (accepted for publication)

[22] J. Narayan, O.S. Oen, D. Fathy and O.W. Holland, Materrials Letters 3 (1985) 67

[23] F. Spaepen, D. Turnbull, Laser Solid Interactions and Laser Processing, ed. S.D. Ferris, H.J. Leamy, J.M. Poate, Mat. Res. Soc., Boston, 1978

[24] G.-W. Lu, E. Nygren, M.J. Aziz, J. Appl. Phys. 70 (1991) 5323 
[25] J.S. Williams, R.G. Elliman, Phys. Rev. Lett. 51 (1983) 1069

[26] F. Priolo, A. Battaglia, R. Nicotra, Appl. Phys. Lett. 57 (1990) 768

[27] L. Pelaz and G.H. Gilmer, private communication

[28] J. Zhu, T. Diaz de la Rubia, L. Yang, C. Mailhiot and G. H. Gilmer, Phys. Rev. B 1996

[29] P.C. Zalm, J. Appl. Phys. 54 (1983) 2660

[30] P. Bedrossian, M-J Caturla, T. Diaz de la Rubia, Appl. Phys. Lett (accepted for publication). 


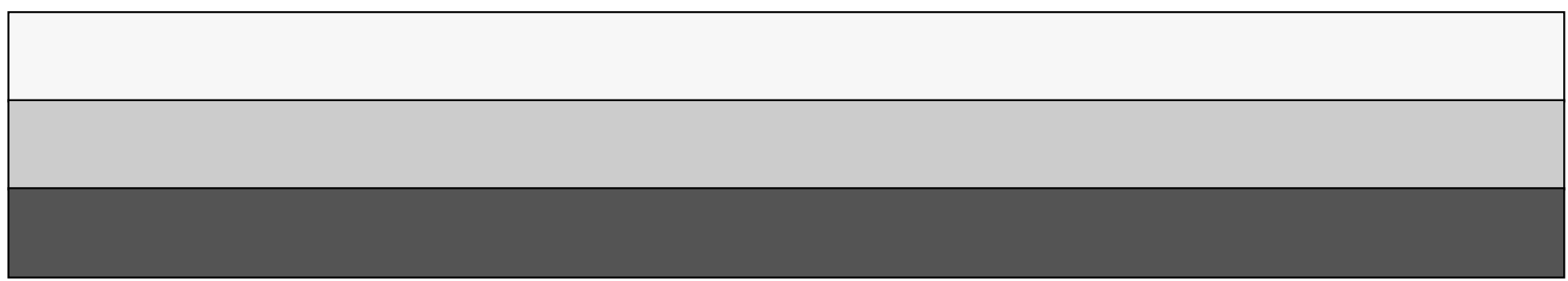

